\title{
MyMap als Werkzeug im GW-Unterricht
}

\author{
*hartwig.hitz@schule.at, BRG Maria Enzersdorf, Pädagogische Hochschule Niederösterreich, Universität Wien \\ **karel.kriz@univie.ac.at, Institut für Geographie und Regionalforschung, Universität Wien, \\ *** alexander.pucher@univie.ac.at, Institut für Geographie und Regionalforschung, Universität Wien
}

Dieser Beitrag sowie das angeführte Unterrichtsbeispiel verfolgen verschiedene Ziele: Das sind die computergestützte Kartenerstellung, die Kartenmanipulation, die Kartenkritik und damit verbunden die Sensibilisierung zu kritischem Kartenlesen in verschiedenen Medien.

\section{Hintergrund und Motivation der Ent- wicklung von MyMap}

Bereits vor mehr als zehn Jahren wurde der Bedarf an einer schnellen und effizienten Möglichkeit zur Erstellung einfacher thematischer Karten von Österreich an das Institut für Geographie und Regionalforschung der Universität Wien herangetragen. Der Einsatz gängiger GIS-Pakete, wie etwa ArcGIS, MicroStation, Intergraph, aber auch Open-Source Produkte wie QGIS oder GRASS GIS, stellen hierfür keinen zufriedenstellenden Ansatz dar. Geographische Informationssysteme sind durch eine hohe Komplexität der Funktionalitäten gekennzeichnet, die weit über die Bedürfnisse der Erstellung kartographischer Darstellungen hinausgeht, und damit eine entsprechend lange Einarbeitung notwendig machen.

Vor diesem Hintergrund wurde am Institut für Geographie und Regionalforschung der Universität Wien die Umsetzung von MyMap in Angriff genommen (siehe dazu Hitz 2006), das mittlerweile in einer völlig überarbeiteten Version zur Verfügung steht: http://exp.geo.univie.ac.at/projects/mymap/public/. MyMap bietet einen einfachen Ansatz zur kartographischen Visualisierung thematischer Sachverhalte. Das System ermöglicht die Erstellung einfacher Choroplethenkarten, insbesondere für den Gebrauch im Unterricht.

Wesentlicher Ansatz war es dabei, die Möglichkeiten der thematisch-kartographischen Bearbeitung der Darstellung auf entsprechend einfache und nachvollziehbare Weise zur Verfügung zu stellen. Diese Einfachheit spiegelt sich auch beim Zugang zu MyMap wider: Das Tool ist ohne Registrierung mit einer Mai- ladresse verfügbar, im Unterricht also sofort für alle Schüler/innen einsetzbar. Um dennoch über mehrere Unterrichtseinheiten hinweg an einem Projekt arbeiten zu können, bietet sich der Export als Textfile an (siehe weiter unten).

Sämtliche thematisch-kartographischen Aspekte der Karten in MyMap können vom Nutzer verändert und angepasst werden.

Folgende Adaptionen der Karten können vorgenommen werden:

- Anzahl der Klassen (2-7)

- Statistische Methode der Klassifizierung (prozentuell, gleicher Abstand)

- Schwellenwerte aller Klassengrenzen

- Unterschiedliche Farbskalen

- Änderung einzelner Klassenfarben

- Alternativtext zur Beschriftung der Klassen

- Änderung sämtlicher Texte (Titel, Untertitel, Legende, Bearbeiter, Datenquelle)

Zur weiteren Verwendung der Karten können diese jederzeit als Grafik (png) abgespeichert und in den Unterricht integriert werden. Darüber hinaus besteht die Möglichkeit, eine Beschreibungsdatei (Textfile) der Karte abzuspeichern, in dem sämtliche Parameter der Bearbeitung enthalten sind. So ist es etwa denkbar, eine Kartenbeschreibung zur späteren Weiterverarbeitung zu sichern, oder eine in Arbeit befindliche Karte an andere Personen zu Bearbeitung zur Verfügung zu stellen.

MyMap stellt aber nicht den Anspruch, ein Atlasähnliches Produkt zu sein. Hierfür soll auf den ÖROK Atlas Online (www.oerok-atlas.at) verwiesen werden, der wohl die umfangreichste Sammlung an thematischen Karten Österreichs darstellt. Die Möglichkeiten, selbst Karten zu bearbeiten oder überhaupt mit eigenen Daten erstellen zu können (siehe dazu Koller \& Hitz 2008) wurde 2014 aus dem ÖROK-Atlas entfernt.

MyMap beinhaltet einige Kartenbeispiele, anhand derer die Funktionsweise geprobt werden kann. Der wesentliche Mehrwert von MyMap ergibt sich allerdings durch die Möglichkeit, eigene thematisch-sta- 


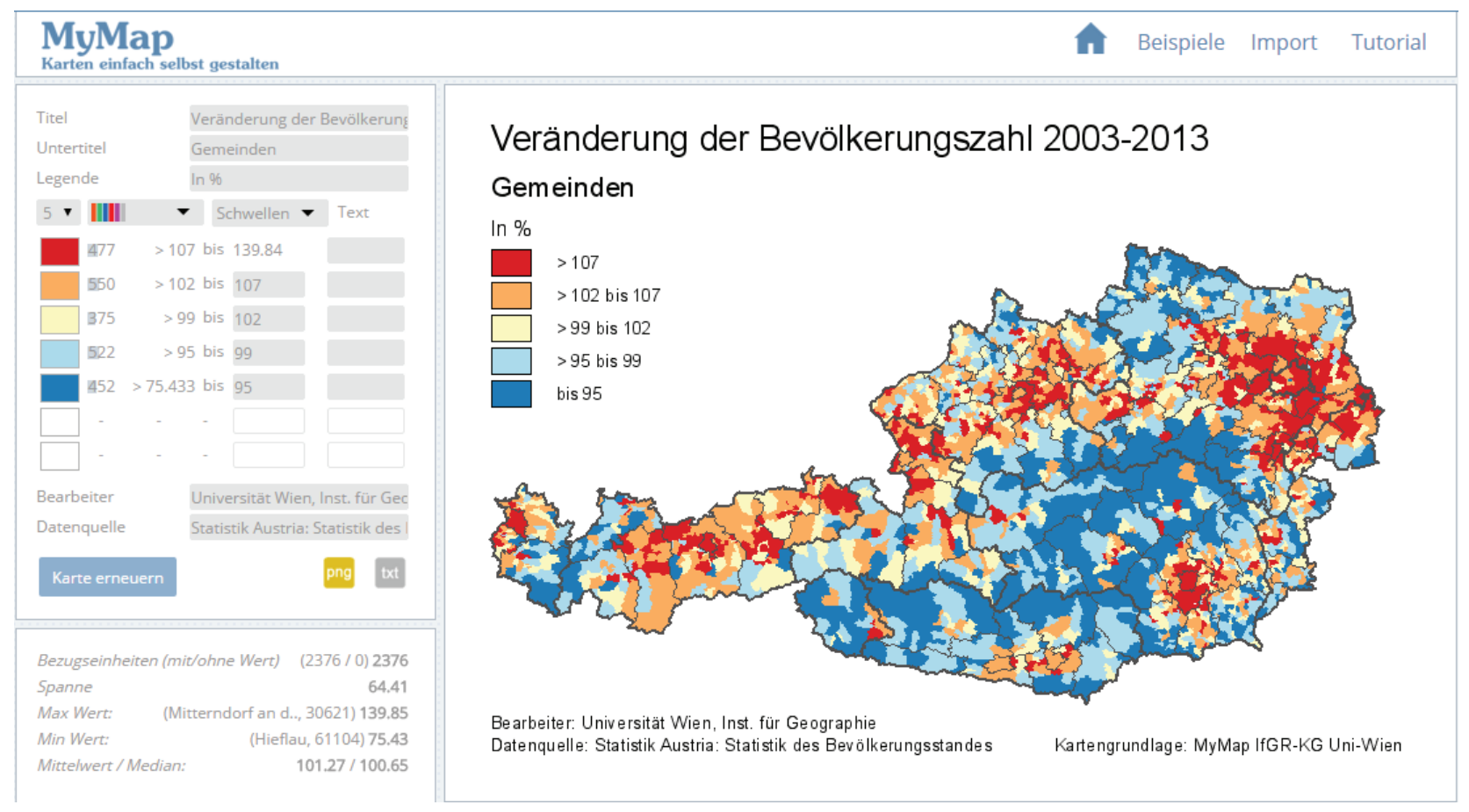

Abb. 1: MyMap-Kartenbeispiel „Veränderung der Bevölkerungszahl 2003-2013“(Quelle: http:/lexp.geo.univie.ac.at/projects/mymap/public/).

tistische Daten integrieren zu können. Dieser „Datenimport" ist derzeit für österreichische Gemeinden und Bezirke, sowie NUTS 3 Gebiete möglich. Mit MyMap steht somit ein Werkzeug zur Verfügung, dass sowohl Lehrer/innen die Möglichkeit bietet, Karten für den Unterricht vorzubereiten, wie auch die Option, diese von Schülerinnen und Schülern umsetzen und gestalten zu lassen.

Ein wesentlicher Kernpunkt des Systems ist die freie Zugänglichkeit über das Internet, die auch kartographisch Ungeübten jederzeit und ortsunabhängig ein qualitativ hochwertiges Werkzeug zur Datenvisualisierung bereitstellt. Die offene Gestaltung und Umsetzung der Applikation mit Open-Source Komponenten gestattet es jedem Interessierten, das System an seine persönlichen Bedürfnisse anzupassen.

Der technische Aufbau von MyMap besteht aus 3 wesentlichen Komponenten. Als Basis dient PostgreSQL als relationales Datenbankmanagementsystem. Der programtechnische Kern von MyMap wurde in PHP umgesetzt. Als graphische Komponente zur Erstellung der Karten dient UMN Mapserver. MyMap ist in jeglicher (technischer) Hinsicht modular konzipiert und umgesetzt worden. Dies ermöglicht die prinzipielle Erweiterung des Systems mit zusätzlichen Funktionalitäten, aktuellen (und damit leistungsfähigeren) Komponenten sowie das Hinzufügen weiterer Basisgeometrien, neben den derzeit verfügbaren österreichischen Gemeinden und Bezirke, sowie NUTS 3 Gebieten.

MyMap wurde von den Mitarbeiterinnen und Mitarbeitern des Instituts für Geographie und Re- gionalforschung der Universität Wien in Eigenregie entwickelt und in unregelmäßigen Abständen weiterentwickelt. Entsprechende Erweiterungen in vielerlei Hinsicht sind denkbar und wünschenswert. Die Universität Wien, Institut für Geographie und Regionalforschung ist in diesem Zusammenhang sehr an der Zusammenarbeit mit interessierten Lehrerinnen und Lehrern interessiert, um MyMap einer breiteren Anwendergruppe im schulischen Bereich bekannt und zugänglich zu machen.

\section{Einsatz im Schulunterricht}

Der Geographie und Wirtschaftskundelehrplan der AHS-Oberstufe fordert sowohl in den Bildungsund Lehraufgaben als auch in den didaktischen Grundsätzen an mehreren Stellen den Einsatz von computergestützten Verfahren bzw. die Nutzung der Möglichkeiten der IKT (RIS 2015a). Anders als im HTL-Lehrplan (RIS 2015b) kommt der Begriff "Geographische Informationssysteme" im AHS-Lehrplan nicht vor. An vielen Schulen fehlt aber ohnehin die Zeit, mit "großen" GIS-Paketen wie ArcGIS oder QGIS zu arbeiten, da die Einarbeitungszeit die produktive Arbeit mit den Schülerinnen und Schülern bei weitem übersteigen würde. Um den Forderungen aus dem AHS-Lehrplan aber dennoch gerecht zu werden, empfiehlt sich der Einsatz webgestützter Informationssysteme wie MyMap.

Auf die eingangs angesprochenen Aufgaben des Unterrichtsfachs Geographie und Wirtschaftskunde 
Tab. 1: Im Lehrplan geforderte Kompetenzen und deren Umsetzung mit MyMap

\begin{tabular}{|c|c|}
\hline $\begin{array}{l}\text { Im Lehrplan geforderte Kompetenzen } \\
\text { (RIS 2015a, Hervorhebung durch die Autoren) }\end{array}$ & Umsetzung mit MyMap \\
\hline $\begin{array}{l}\text { Methodenkompetenz: geographisch-wirtschaftskundliche } \\
\text { Informationen mit Hilfe bewährter und auch mit dem Ein- } \\
\text { satz computergestützter Verfahren gewinnen, analysie- } \\
\text { ren und zielgruppenorientiert darstellen können }\end{array}$ & $\begin{array}{l}\text { Die Darstellung von geographisch-wirtschaftskundlichen } \\
\text { Informationen kann auf zwei Arten erfolgen - in Diagram- } \\
\text { men oder thematischen Karten. Die geforderte ziel- } \\
\text { gruppenorientierte Darstellung lässt sich mit MyMap mit } \\
\text { einfachen Flächenkartogrammen einfach realisieren. }\end{array}$ \\
\hline $\begin{array}{l}\text { Gesellschaftskompetenz: die Fähigkeit erweitern, die von } \\
\text { den Massenmedien verbreiteten politischen, wirt- } \\
\text { schaftlichen und gesellschaftlichen Informationen über } \\
\text { Österreich, Europa und die Welt kritisch zu beurteilen. }\end{array}$ & $\begin{array}{l}\text { Durch die Möglichkeit, selbst die Zahl der Klassen als auch } \\
\text { der Schwellenwerte festzulegen, können die Schüler/innen } \\
\text { mit ein und demselben Datenmaterial sehr schnell ver- } \\
\text { schiedene Karten erzeugen, die unterschiedliche Interpreta- } \\
\text { tionen zulassen und somit den Schülerinnen und Schülern } \\
\text { vor Augen führen können, wie auch in Massenmedien Kar- } \\
\text { ten gestaltet werden können und daher kritisch betrachtet } \\
\text { werden müssen. Siehe dazu auch das Unterrichtsbeispiel. }\end{array}$ \\
\hline
\end{tabular}

Tab. 2: Didaktische Grundsätze und deren Umsetzung mit MyMap

\section{Didaktische Grundsätze (RIS 2015a, Hervorhebung durch die Autoren)}

Im Unterricht soll die Aktivität der Schüler/innen im Vordergrund stehen. Daher sind verstärkt Unterrichtsverfahren einzusetzen, die zu eigenständiger und kritischer Informationsverarbeitung führen. Dabei sind neben traditionellen geographischen Arbeitsformen insbesondere die Möglichkeiten der IKT zur Gewinnung sowie Verarbeitung und Darstellung geographischer und wirtschaftskundlicher Informationen zu nutzen.

\section{Umsetzung mit MyMap}

Hier gilt Ähnliches wie bei der Methodenkompetenz. Beim Einsatz der mannigfaltigen Möglichkeiten der IKT zur Darstellung geographischer und wirtschaftlicher Informationen bieten sich diverse Textdarstellungen, Diagramme und eben Karten an. in der Oberstufe wird in Tabelle $1 \& 2$ näher eingegangen.

Über den möglichen Einsatz von selbst erstellten Karten im Rahmen der mündlichen Reifeprüfung hat Sitte 2006 bereits geschrieben. Mit MyMap steht nun ein mächtiges Werkzeug zur Verfügung, das auch im Rahmen der kompetenzorientierten mündlichen Reifeprüfung verwendet werden kann.

\section{Anmerkungen zum Unterrichtsbeispiel}

Das Unterrichtsbeispiel zum Thema „Ausländischer Staatsangehörige in Österreich", wurde im Herbst 2014 vom Autor in einer 7. Klasse praktisch erprobt. Obwohl die Schüler/innen in der 5. Klasse im Pflichtfach Informatik den Umgang mit einem Tabellenkalkulationsprogramm erlernt haben und der Lehrplan für Mathematik in der Oberstufe „Tabellenkalkulationsprogramme (...) im heutigen Mathematikunterricht (für) unverzichtbar“ (RIS 2015a) ansieht, stellten die Vorarbeiten der Datenaufbereitung für viele Schüler/innen große Probleme dar. Dies sollte aber kein Grund sein, den Computer im GWK-Unterricht nicht einzusetzen, sondern viel mehr als Auftrag ge- sehen werden, den im Lehrplan geforderten Einsatz von computergestützten Verfahren bzw. die Nutzung der Möglichkeiten der IKT im GWK-Unterricht zu forcieren. Auch die letzten beiden Aufgaben (Interpretation und bewusste Kartenmanipulation) sind eine Herausforderung für manche Schüler/innen, aber der damit verbundene Beitrag zur Gesellschaftskompetenz darf nicht außer Acht gelassen werden.

Das Arbeitsblatt zum Unterrichtsbeispiel befindet sich im Anhang. Will man die entsprechenden Vorarbeiten zum Besorgen der Daten und zur Datenaufbereitung umgehen, um den Fokus stärker auf das Erstellen, Verändern und Interpretieren der Karte zu richten, können die Punkte 1 bis 12 von der Lehrperson vorbereitet werden und die Schüler/innen können direkt mit der Erstellung der Karte unter Punkt 13 beginnen. Die Daten für dieses Unterrichtsbeispiel finden sich auch bei den Kartenbeispielen auf MyMap, womit der komplette Prozess der Datenaufbereitung und des Datenimport eingespart werden kann. Es ist zwingend notwendig, spätestens mit diesem Punkt zu beginnen, da nur so die Schüler/innen die Möglichkeit haben, Schwellenwerte zu ändern und vollkommen eigene Karten zu gestalten. 


\section{$4 \quad$ Literatur}

Hitz, H. (2006): MyMap - Thematic Mapping Tool. In: GW-Unterricht 102, 95-97.

Koller, A. \& H. Hitz (2008): Thematische Karten - visualisiert in Google Earth. Ein Unterrichtsbeispiel mit fachdidaktischer Analyse. In: GW-Unterricht 114, 51-55.

RIS - Rechtsinformationssystem des Bundes (2015a): Verordnung des Bundesministers für Unterricht und Kunst vom 14. November 1984 über die Lehrpläne der allgemeinbildenden höheren Schulen https:/www.ris.bka. gv.at/GeltendeFassung.wxe?Abfrage=Bundesnormen $\& \mathrm{G}$ esetzesnummer $=10008568(10.04 .2015)$.
RIS - Rechtsinformationssystem des Bundes (2015b): Verordnung der Bundesministerin für Unterricht, Kunst und Kultur über die Lehrpläne der Höheren technischen und gewerblichen Lehranstalten (Lehrplan 2011) https:// www.ris.bka.gv.at/GeltendeFassung.wxe?Abfrage=Bund esnormen\&Gesetzesnummer=20007451 (10.04.2015).

Sitte, C. (2006): Der ÖROK-Atlas - www.oerok-atlas.at. Ein neues, IKT-gestütztes online verfügbares Hilfsmittel für den GWK-Unterricht. In: Wissenschaftliche Nachrichten 131, 47-50. 


\section{Anteil ausländischer Staatsangehöriger in den Bezirken Österreichs 2011}

\section{Erstellen einer thematischen Karte mit MyMap}

1. Gehe auf die Website der Statistik Austria (www.statistik.at) und wähle den Menüpunkt „Bevölkerung“ und anschließend „Volkszählungen, Registerzählung, Abgestimmte Erwerbsstatistik“.

2. Lade dir nun die Datei „Registerzählung 2011: Gemeindetabelle Österreich“ im xls-Format auf dein Laufwerk herunter (Direktlink: www.statistik.at/web_de/static/registerzaehlung_2011_gemeindetabelle_ oesterreich_073982.xlsx).

3. Gehe auf www.univie.ac.at/cartography/mymap - unter Tutorial findest du Excelvorlagen für die verschiedenen Raumeinheiten. Lade dir die Vorlagen für die Bezirke und Gemeinden herunter und öffne sie.

4. Du wirst bemerken, dass der Datensatz der Statistik Austria so nicht im MyMap verwendet werden kann dazu sind noch Vorarbeiten notwendig.

\section{Vorarbeiten}

5. Entferne im Datensatz der Statistik Austria alle verbundenen Zeilen (Zeilen 1, 3, 4 und 2478).

6. Kopiere nun die drei Spalten, die laut Beispieldatei benötigt werden (Regionale Kennziffer; Bundesland, Politischer Bezirk, Gemeinde; Anteil ausländischer Staatsangehöriger), und füge sie in einer neuen Datei ein.

7. Der Datensatz sollte nun folgendermaßen aussehen und über 2474 Zeilen verfügen.

\begin{tabular}{|c|c|c|c|}
\hline & $A$ & B & C \\
\hline 1 & 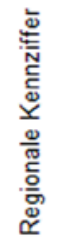 & $\begin{array}{l}\text { Bundesland } \\
\text { Politischer Bezirk } \\
\text { Gemeinde }\end{array}$ & 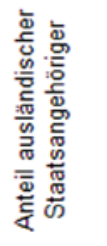 \\
\hline 2 & 0 & Österreich & 11,2 \\
\hline 3 & & 1 Burgenland & 6,0 \\
\hline 4 & & 1 Eisenstadt (Stadt) & 9,4 \\
\hline 5 & & 2 Rust (Stadt) & 4,7 \\
\hline 6 & & 3 Eisenstadt-Umgebung & 5,9 \\
\hline 7 & 103 & 1 Breitenbrunn am Neusiedler See & 5,6 \\
\hline 8 & 103 & 2 Donnerskirchen & 3,8 \\
\hline
\end{tabular}

8. Da wir den Anteil ausländischer Staatsangehöriger in den Bezirken Österreichs 2011 darstellen möchten, können wir die Gemeinden und Bundesländer - mit Ausnahme von Wien - löschen. Sortiere dazu am besten die gesamte Tabelle nach Spalte A und lösche anschließend die nicht benötigten Zeilen.

9. Damit Wien dargestellt werden kann, musst du bei Wien die „Regionale Kennziffer“ von 9 auf 900 ändern. 
10. Tausche nun die Spalten B und C, sodass „Anteil der ausländischen Staatsangehörigen“ und die zugehörigen Werte in Spalte B sowie „Bundesland, Politischer Bezirk, Gemeinde“ und die zugehörigen Namen in Spalte $\mathrm{C}$ stehen.

\begin{tabular}{|c|c|c|c|}
\hline & A & B & c \\
\hline & 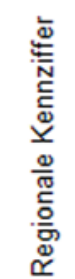 & 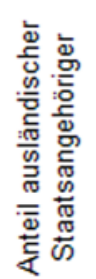 & $\begin{array}{l}\text { Bundesland } \\
\text { Politischer Bezirk } \\
\text { Gemeinde }\end{array}$ \\
\hline 2 & 900 & \multicolumn{2}{|c|}{21,7 Wien } \\
\hline 3 & 101 & \multicolumn{2}{|c|}{ 9,4 Eisenstadt (Stadt) } \\
\hline 4 & 102 & \multicolumn{2}{|c|}{ 4,7 Rust (Stadt) } \\
\hline
\end{tabular}

11. Zum Abschluss musst du die Datei noch im csv-Format speichern. Wähle dafür den Dateityp „Text CSV (.csv) (*.csv)“ und aktiviere das Kästchen bei „Filtereinstellungen bearbeiten“. Wähle als Feldtrenner den Strichpunkt; und als Texttrenner kein Zeichen aus.

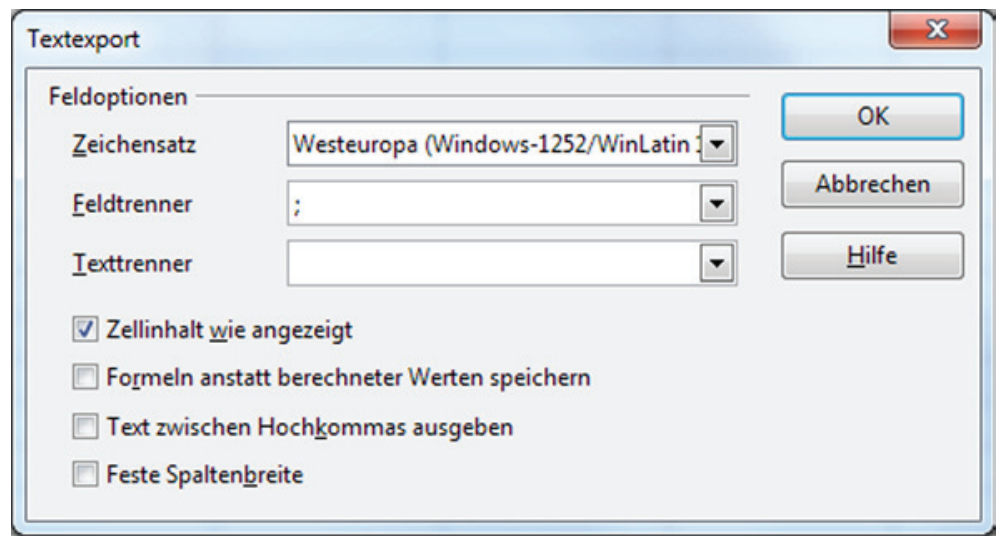

\section{Erstellung der Karte in MyMap}

12. Ändere die Dateiendung von *.csv auf*.txt um.

13. Wenn du möchtest, kannst du die csv-Datei nun mit dem Editor bearbeiten und gleich dort Titel, Untertitel, Bearbeiter usw. eintragen. Der Vorteil ist, dass diese Daten dann automatisch in die Legende und Karte übernommen werden. Füge dazu am Ende der Datei folgende Zeilen ein:

- Titel: Anteil ausländischer Staatsangehöriger

- Untertitel: in den Bezirken Österreichs 2011

- Bearbeiter: dein Name

- Quelle: Statistik Austria

- Legendentext: in Prozent

- Farbskala: rot

14. Gehe nun auf www.univie.ac.at/cartography/mymap und wähle dort „eigene Daten importieren“ aus.

15. Mit „Datei auswählen“ kannst du nun deine txt-Datei suchen und auswählen und mit der Schaltfläche „Abschicken" erstellst du deine Karte. 
16. Solltest du unter Punkt 12 keine Legendentexte und keinen Kartentitel eingetragen haben, kannst du dies jetzt machen.

17. Speichere anschließend die Karte mit einem Klick auf png.

\section{Interpretation / Kritik}

18. Interpretiere die von dir erstellte Karte! Was kannst du aus dieser Karte herauslesen? Wo ist der Anteil ausländischer Staatsangehöriger höher, wo niedriger? Was sind die Gründe dafür?

- Spezialfrage zu den aktuellen Daten in der Steiermark: Überlege, warum in der Karte in der Steiermark Flächen weiß geblieben sind? Zur Beantwortung der Frage kannst du auch im Internet recherchieren!

19. Experimentiere nun mit den Schwellen - verwende die Schwellenmöglichkeiten, die dir MyMap bietet, versuche aber auch eigene Schwellenwerte. Erstelle eine Karte, die für die auflagenstärkste österreichische Tageszeitung geeignet wäre, um zu zeigen, dass in Österreich viele ausländische Staatsangehörige leben. Finde zuerst heraus, welche Ideologie diese Tageszeitung verfolgt. Wenn du damit zufrieden bist bzw. der Chefredakteur dieser Tageszeitung zufrieden wäre, speichere diese Karte ebenfalls! 